\title{
El modelo argumentativo de Toulmin y la educación en ciencias: una revisión argumentada
}

\section{Toulmin's argumentative model and science education: an argued review}

Jorge Pinochet ${ }^{1}$

\begin{abstract}
Resumen: En el presente trabajo se revisa críticamente la investigación que ha empleado el modelo de Toulmin como marco teórico para estudiar la argumentación en las clases de ciencias. Para ello se pone especial énfasis en revisar la literatura indexada, presentando los principales resultados de los estudios, y también sus alcances y limitaciones. Se identifican algunas tendencias, y se discute la relevancia educativa de la investigación sobre el tema, reflexionando acerca de diversas problemáticas que emergen en este campo. Finalmente, se examina la literatura en su conjunto, identificando problemas abiertos que ofrecen oportunidades para emprender futuras investigaciones.
\end{abstract}

Palabras clave: Modelo de Toulmin. Argumento. Educación en ciencias. Investigación. Enseñanza de las ciencias.

\begin{abstract}
This paper critically reviews the research that has used Toulmin's model as a theoretical framework to study reasoning in science classes. Special emphasis is placed on reviewing the indexed literature, presenting the main findings of the studies, their scope and limitations. Some trends are identified, and the educational significance of the research on the subject is discussed. Various issues that emerge in this field are also analyzed. Finally, the literature as a whole is reviewed, identifying unsolved matters that offer opportunities for future research.
\end{abstract}

Keywords: Toulmin Model. Argument. Science education. Research. Science teaching.

\footnotetext{
${ }^{1}$ Facultad de Educación, Universidad Alberto Hurtado (UAH), Almirante Barroso 37, Santiago Centro, Chile. E-mail: <jpinochet@uahurtado.cl>
} 


\section{Introducción}

El estudio de la argumentación en los procesos de enseñanza y aprendizaje de las ciencias constituye una línea de investigación que ha experimentado un crecimiento significativo durante las últimas dos décadas, tanto por la cantidad como por la diversidad de las temáticas abordadas. Estos estudios han sido profundamente influidos por el modelo argumentativo desarrollado por el filósofo británico Stephen Toulmin (1958), pues un amplio porcentaje de las investigaciones se basan en dicho modelo, ya sea porque se han inspirado en él, o bien porque lo han empleado como marco teórico para describir y analizar el discurso de estudiantes y profesores de ciencias.

La presente revisión bibliográfica tiene como objetivo examinar críticamente la investigación educativa que ha estudiado los procesos de argumentación en las clases de ciencias en base al modelo de Toulmin. Para ello, se comienza describiendo brevemente la metodología empleada en la revisión. Luego, se evalúan aspectos generales del modelo de Toulmin, tales como sus características básicas y sus distintos componentes. Después se discuten los alcances y limitaciones de diversas investigaciones indexadas que han usado este modelo, así como sus metodologías. También se exploran algunas líneas de investigación que han emergido en este campo, y se discuten diferentes propuestas que han pretendido modificar o enriquecer el modelo del filósofo británico. Para finalizar, se analizan algunos problemas abiertos que ofrecen oportunidades para emprender futuras investigaciones.

\section{Metodología de la revisión}

Luego de examinar distintas bases de datos ${ }^{2}$, se constató que la gran mayoría de la investigación sobre argumentación en las clases de ciencias se encuentra alojada en la ISI Web of Knowledge ${ }^{3}$, de modo que la revisión bibliográfica se centró en esta base. En particular, se trabajó con las bases Science Citation Index Expanded (SCI-EXPANDED) y con Social Sciences Citation Index (SSCI), focalizando la búsqueda en los "Articles" y en los "Proceedings papers", que reúnen la mayor parte de la literatura pertinente para esta revisión.

Para identificar la bibliografía de interés, se comenzó utilizando como términos de búsqueda “Toulmin", “argument*” y "scien*” en todos los años disponibles (1988-2011), empleando criterios de filtro que condujeran específicamente a trabajos relacionados con la educación en ciencias. Al utilizar estos términos en la modalidad de búsqueda combinada, mediante el operador booleano AND, se desplegaron tan sólo 24 trabajos, de los cuales 15 cumplieron con los criterios de inclusión. Por esta razón, se realizó una segunda búsqueda análoga a la anterior, pero menos restrictiva, incluyendo únicamente lo términos "argument*" y "scien*". En estas condiciones, aparecieron 366 artículos que fueron filtrados atendiendo a tres criterios básicos: nombre de la publicación; título del trabajo; resumen o abstract. Cuando los criterios anteriores resultaron insuficientes o dejaron algún margen de duda, fue necesario revisar el contenido de

\footnotetext{
${ }^{2}$ Scielo (disponible en: <http://www.scielo.org/php/index.php>. Acceso el: 23 jan. 2015), Latindex (disponible en: <http://www.latindex.unam.mx/>. Acceso el: 23 enero 2015) y otras.

${ }^{3}$ Disponible en: <http://www.webofknowledge.com/>. Acceso el: 23 enero 2015).
} 
las publicaciones. $\mathrm{Al}$ proceder de este modo, el número de artículos se redujo a 83 , que fue la cantidad seleccionada en forma definitiva. Otro criterio empleado en la revisión fue el número de referencias de las publicaciones, así como el número de citas de cada autor, pues ello permitió identificar las investigaciones y los autores más significativos. Es importante agregar que un amplio porcentaje de los trabajos seleccionados comprenden la década 2000-2010, de modo que la revisión se centró fundamentalmente en este periodo.

La revisión bibliográfica no se limitó a examinar la literatura indexada, pues una parte del material fue identificado mediante las referencias incorporadas al final de los artículos escogidos. En este caso, el criterio de inclusión consistió básicamente en considerar la importancia concedida a cada referencia en las publicaciones donde fueron citadas. Después de la identificación y selección de los artículos que cumplieron con los criterios de inclusión, se procedió a analizarlos, buscando similitudes, diferencias, patrones y tensiones que permitieran articular este trabajo.

\section{El modelo argumentativo de Toulmin}

\section{Aspectos generales}

Los primeros antecedentes de la investigación educativa sobre argumentación datan de mediados del siglo XX, cuando algunos autores comenzaron a plantear severas críticas a la lógica formal. Desde los tiempos de su fundador, el filósofo griego Aristóteles, la lógica había pretendido convertirse en una ciencia deductiva comparable a la matemática. Sin embargo, tal como han sostenido sus críticos, la tradición aristotélica ha supuesto una enorme simplificación respecto de la riqueza y complejidad del lenguaje ordinario. Las críticas más importantes a la lógica formal surgieron de los trabajos seminales de Stephen Toulmin (TOULMIN, 1958; TOULMIN; RIEKE; JANIK, 1979) y Chaïm Perelman (PERELMAN; OLBRECHTS-TYTECA, 1958), quienes, además de dar origen a la teoría moderna de la argumentación, pueden considerarse los principales impulsores del movimiento de lógica informal, que significó una ruptura definitiva con la tradición aristotélica, al desplazar el interés hacia la retórica, el lenguaje natural y la argumentación dialógica.

Es precisamente dentro de la perspectiva de la lógica informal donde se inscribe la investigación educativa inspirada en el modelo de Toulmin. Desde un comienzo, la mayor parte de dicha investigación se ha focalizado en examinar el discurso que se desarrolla en las clases de ciencias pues, como han reconocido diversos autores, la argumentación ocupa un lugar central en la actividad científica. En efecto, la ciencia produce principalmente explicaciones acerca de cómo o porqué ocurre un determinado fenómeno, y estas explicaciones son construidas, evaluadas y comunicadas a través de la argumentación (JIMÉNEZ-ALEIXANDRE; BUGALLO RODRÍGUEZ; DUSCHL, 2000; LAWSON, 2003; MCNEILL; KRAJCIK, 2009). La perspectiva que concibe el aprendizaje de las ciencias como argumentación fue propuesta hace dos décadas por Deanna Kuhn (1992, 1993). Desde aquella época, diversos autores (por ejemplo, DRIVER; NEWTON; OSBORNE, 2000) han sugerido que la educación científica debe promover la argumentación como una dimensión fundamental del aprendizaje de las ciencias.

El modelo de Toulmin ha resultado fecundo, tanto dentro como fuera del campo de la educación (ver Figura 1). En el terreno específico de la investigación educativa, además de 
sus importantes aplicaciones en el estudio de la argumentación en las clases de ciencias, el modelo ha sido usado en ámbitos tan diversos como la matemática (KRUMMHEUER, 1995), la historia (PONTECORVO; GIRARDET, 1993), el Inglés (MITCHELL, 1996), e incluso como herramienta heurística para evaluar el trabajo de los estudiantes (HART, 1998). Sin embargo, esta versatilidad no es privativa del modelo de Toulmin, pues existen marcos teóricos alternativos que también permiten estudiar la argumentación en distintos espacios educativos, tanto científicos como no científicos. Tal es el caso del modelo propuesto por Schwarz et al. (2003) y del Scheme for Presumptive Reasoning elaborado por Walton (1996). Sin embargo, el impacto de estos modelos sobre la investigación educativa, en especial en el dominio de las ciencias, ha sido más bien reducido si se les compara con el esquema de Toulmin.

Figura 1. Una visión esquemática del modelo de Toulmin y sus diversas aplicaciones

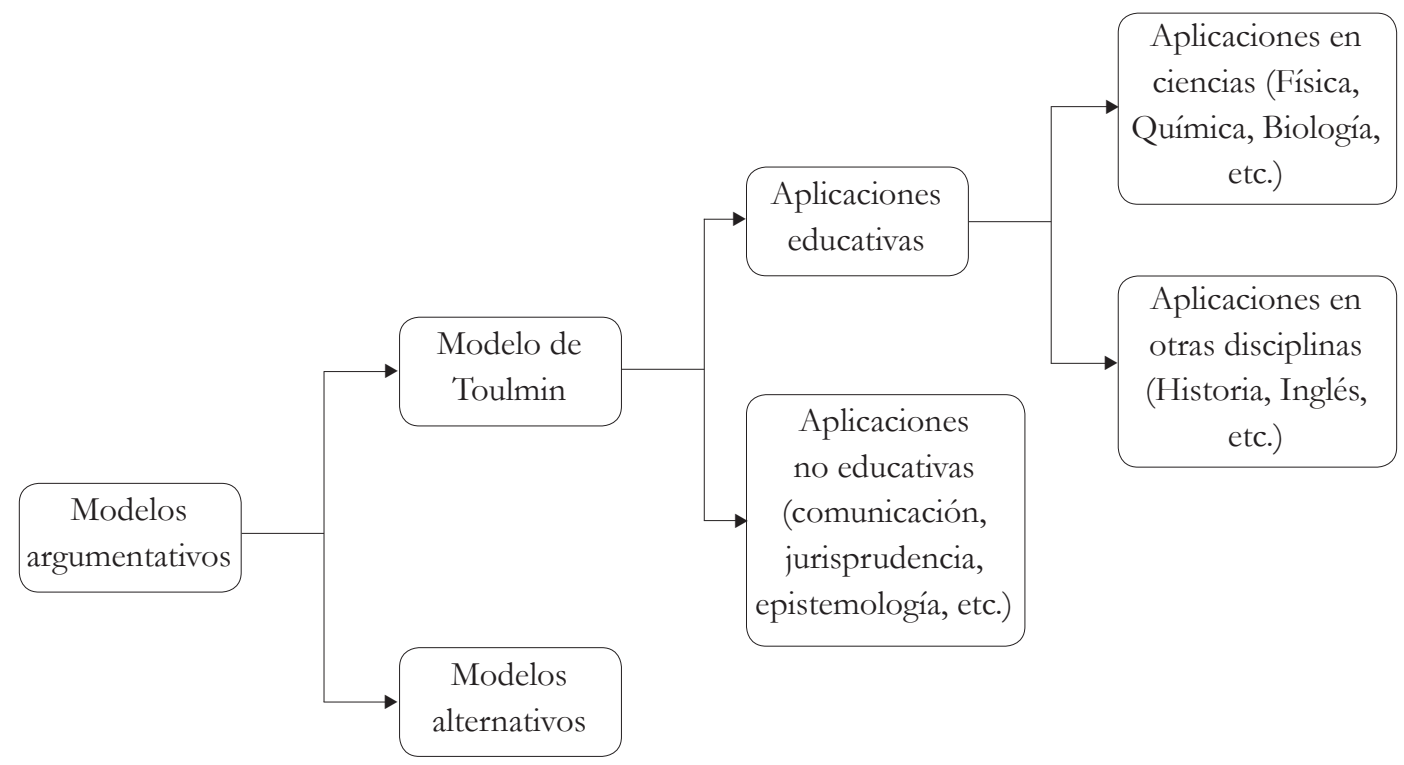

Fuente: elaboración del autor.

\section{El modelo de Toulmin y los argumentos sustantivos}

Antes de discutir en detalle el modelo de Toulmin, resulta pertinente introducir las nociones de argumento y argumentación que se emplearán en esta revisión: un argumento se refiere a los discursos que un estudiante o un grupo de estudiantes producen cuando deben articular o justificar sus conclusiones o explicaciones, mientras que la argumentación alude al proceso de elaboración de esos discursos (OSBORNE; ERDURAN; SIMON, 2004). Estas definiciones 
pueden considerarse una suerte de interpretación educativa de los planteamientos originales de Toulmin, pues aun cuando el filósofo británico nunca hizo alusión a posibles aplicaciones educacionales de su modelo, en lo sustantivo las nociones recién introducidas coinciden con la perspectiva de Toulmin. Además, para los propósitos del presente trabajo, las definiciones propuestas resultan adecuadas porque sintetizan los aspectos generales del proceso argumentativo, tal como es entendido en la literatura sobre educación en ciencias.

Uno de los aspectos más interesantes del modelo de Toulmin - designado habitualmente como TAP por las siglas en inglés de Toulmin's argument pattern - es que ofrece un potente enfoque para estudiar lo que el filósofo británico denomina argumentos sustantivos, es decir, aquellos que deben ser examinados atendiendo a su contenido, lo cual marca una profunda diferencia con la tradición aristotélica, que se interesa únicamente por la forma o estructura de un argumento (en adelante, se entenderá que la expresión TAP hace alusión al modelo de Toulmin aplicado al caso específico de la educación en ciencias).

Toulmin propone que un argumento sustantivo va desde los datos $(D)$ a la conclusión $(C)$, donde $D$ corresponde a la información, antecedentes o hechos de los cuales disponemos para dar fundamento a $C^{4}$. TAP también incorpora la garantía $(G)$, el sustento $(S)$, el calificador modal $(Q)$ y las condiciones de refutación $(R)$. Además, Toulmin supone que un argumento propiamente dicho consiste en al menos tres componentes esenciales: $D, C$ y $G$. La Figura 2 presenta una descripción esquemática de TAP aplicada a un ejemplo concreto acerca del Péndulo de Foucault, exhibido en numerosos museos a través del mundo, y cuyo funcionamiento permite concluir que nuestro planeta gira sobre su propio eje. Naturalmente, un argumento puede volverse bastante más complejo, e incluir varios datos, garantías, refutaciones, etc., pero el esquema de la Figura 2 muestra todos los componentes fundamentales.

Las garantías (warrants) son las que permiten justificar el paso desde los datos (data) a la conclusión (claims), vale decir, $G$ cumple la función de mostrar que el paso de $D$ hacia $C$ es adecuado y legítimo. El modelo incorpora explícitamente el grado de certeza (o incerteza) del argumento mediante el calificador modal Q (qualifier). Ejemplos de calificadores modales son expresiones como: siempre, a veces, probablemente, depende, etc. Además, TAP introduce condiciones de refutación (rebuttals) que establecen las restricciones que se aplican a $C$, es decir, las situaciones bajo las cuales $C$ no sería válida. Finalmente, el sustento (backings) se refiere a las circunstancias generales bajo las cuales $G$ es apoyada.

De acuerdo al modelo de Toulmin, $D, Q, C, G, R$ y $S$ son elementos que no dependen del campo de discurso. Esto hace que TAP sea muy adecuado para analizar las características genéricas de un argumento, pues presenta una estructura que es aplicable en cualquier contexto. Sin embargo, qué cuenta como $D, Q, C, G, R$ y $S$ en un caso particular, es algo que dependiente del campo de discurso. La flexibilidad de TAP para operar tanto en contextos dependientes como independientes del campo, constituye una de sus ventajas para estudiar los argumentos desarrollados por los estudiantes en las clases de ciencias (JIMÉNEZ-ALEIXANDRE, BUGALLO RODRÍGUEZ; DUSCHL, 2000).

\footnotetext{
${ }^{4}$ Para una discusión detallada, consultar en Toulmin (1958); para un tratamiento menos formal ver Toulmin, Rieke y Janik (1979).
} 
Figura 2. Argumento que concluye que la Tierra gira sobre su propio eje, a partir de los datos proporcionados por el péndulo de Foucault

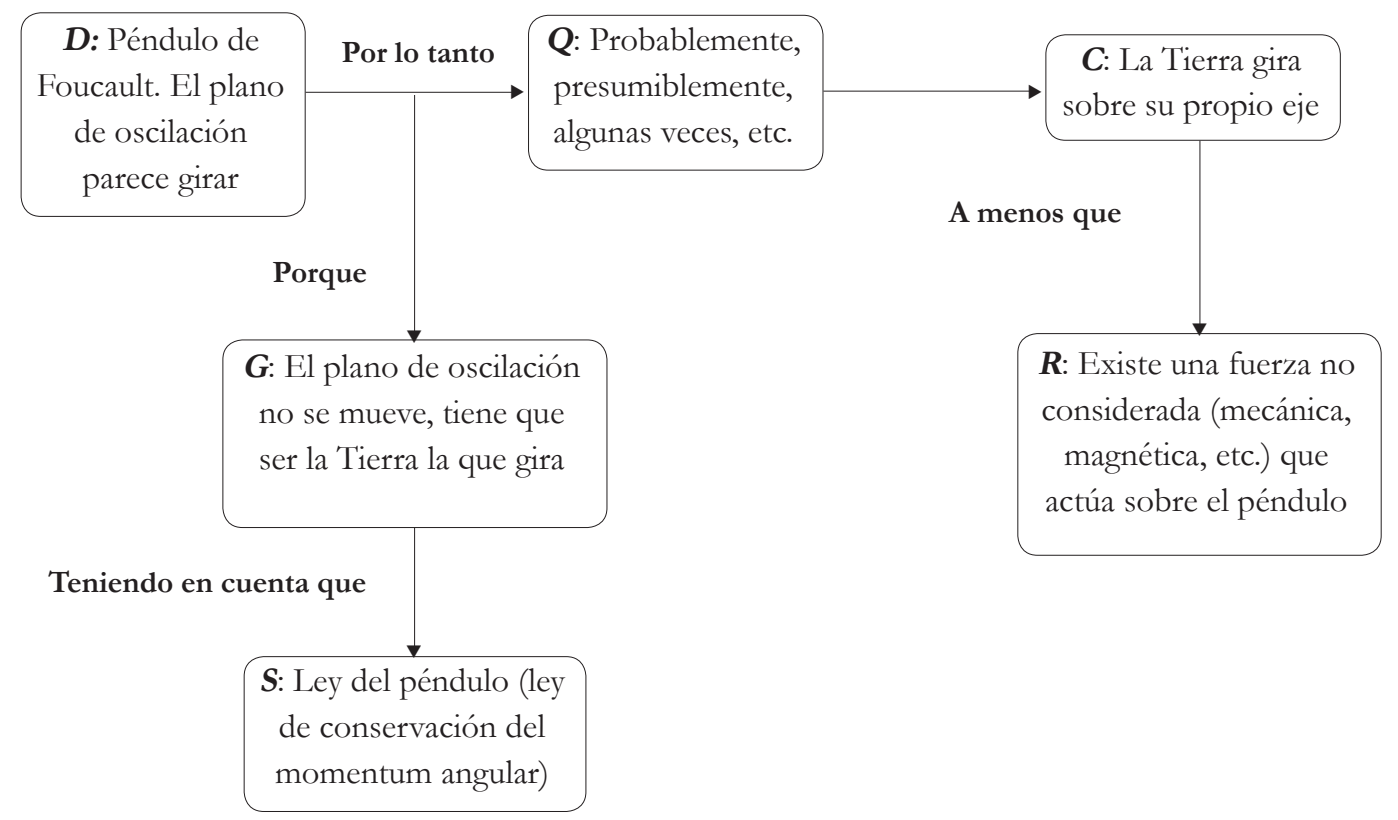

Fuente: adaptado de Jiménez-Aleixandre (2010).

\section{La investigación basada en TAP: una visión panorámica}

\section{Alcances de la investigación}

La investigación basada en TAP ha realizado diversos hallazgos de gran interés tanto para investigadores como para profesores de ciencias. Por ejemplo, Bell y Linn (2000), analizaron los argumentos producidos por estudiantes que pretendían explicar la naturaleza y propagación de la luz. Los autores encontraron que los estudiantes tienden a basarse en los datos para apoyar sus conclusiones, pero con frecuencia no incluyen garantías o sustentos. De forma similar, Jiménez-Aleixandre, Bugallo Rodríguez y Duschl (2000) examinaron la capacidad de un grupo de estudiantes para desarrollar y evaluar argumentos en el contexto de una unidad sobre genética, descubriendo que los argumentos de los estudiantes presentan un predominio de las conclusiones en desmedro de las justificaciones o garantías, las que muestran una baja frecuencia de aparición. En el Cuadro 1 se resumen las características generales y los principales resultados de algunas de las investigaciones más citadas (para otros trabajos que se cuentan entre los más citados, ver, por ejemplo, CLARK; SAMPSON, 2007; JIMÉNEZ-ALEIXANDRE, 2002; VON AUFSCHNAITER et al., 2008). 
Cuadro 1. Principales resultados en las investigaciones más citadas que han empleado TAP

\begin{tabular}{|c|c|c|}
\hline $\begin{array}{l}\text { Autores de la } \\
\text { investigación }\end{array}$ & Características & Principales resultados \\
\hline Bell; Linn (2000) & $\begin{array}{l}\text { Investigación realizada con apoyo de } \\
\text { Internet, que provee una plataforma } \\
\text { para el trabajo de los estudiantes. Se } \\
\text { evalúa la construcción de argumentos } \\
\text { por parte de los estudiantes, acerca de } \\
\text { la naturaleza y propagación de la luz. }\end{array}$ & $\begin{array}{l}\text { Los estudiantes tienden a basarse en } \\
\text { los datos para apoyar sus conclusiones, } \\
\text { pero con frecuencia no incluyen } \\
\text { garantías o sustentos. }\end{array}$ \\
\hline $\begin{array}{l}\text { Jiménez- } \\
\text { Aleixandre; } \\
\text { Bugallo } \\
\text { Rodríguez; } \\
\text { Duschl (2000) }\end{array}$ & $\begin{array}{l}\text { Investigación que examina la capacidad } \\
\text { de un grupo de estudiantes para } \\
\text { desarrollar y evaluar argumentos en el } \\
\text { contexto de una unidad sobre genética. }\end{array}$ & $\begin{array}{l}\text { En argumentos construidos y co- } \\
\text { construidos por estudiantes, se observa } \\
\text { un predominio de las conclusiones } \\
\text { en desmedro de las justificaciones } \\
\text { o garantías, que muestran una baja } \\
\text { frecuencia de aparición. }\end{array}$ \\
\hline $\begin{array}{l}\text { Osborne; } \\
\text { Erduran; Simon } \\
\quad(2004)\end{array}$ & $\begin{array}{l}\text { Investigación que trabajó con un } \\
\text { grupo de profesores de ciencias } \\
\text { que son capacitados en el uso de } \\
\text { la argumentación en aula, y que } \\
\text { luego emplean lo aprendido con sus } \\
\text { estudiantes. También se desarrolla un } \\
\text { marco para evaluar la naturaleza del } \\
\text { discurso argumentativo. }\end{array}$ & $\begin{array}{l}\text { La mayoría de los profesores mejoran } \\
\text { de manera importante sus niveles de } \\
\text { argumentación, lo cual se traduce } \\
\text { en una mejora en las capacidades } \\
\text { argumentativas de sus estudiantes. }\end{array}$ \\
\hline $\begin{array}{l}\text { Kelly; Druker; } \\
\text { Chen (1998) }\end{array}$ & $\begin{array}{l}\text { Investigación que describe un conjunto } \\
\text { de procedimientos metodológicos para } \\
\text { analizar los argumentos de estudiantes } \\
\text { enfrentados a la tarea de resolver } \\
\text { problemas sobre circuitos eléctricos. }\end{array}$ & $\begin{array}{l}\text { Los estudiantes pueden completar la } \\
\text { tarea sin emplear garantías en todos } \\
\text { los argumentos, es decir, el número de } \\
\text { argumentos con garantías producidos } \\
\text { por los estudiantes fue bastante menor } \\
\text { que el número total de argumentos. }\end{array}$ \\
\hline $\begin{array}{l}\text { Simon; Erduran; } \\
\text { Osborne (2006) }\end{array}$ & $\begin{array}{l}\text { Investigación que profundiza en el } \\
\text { trabajo de Osborne y otros (2004) } \\
\text { respecto de la capacitación de los } \\
\text { profesores de ciencias en el uso de la } \\
\text { argumentación. }\end{array}$ & $\begin{array}{l}\text { Los patrones de argumentación y los } \\
\text { cambios operados en los profesores } \\
\text { son específicos de cada uno de ellos. } \\
\text { Además, la mayoría de los profesores } \\
\text { presenta mejoras significativas en sus } \\
\text { niveles de argumentación. }\end{array}$ \\
\hline
\end{tabular}

Fuente: elaboración del autor.

Los resultados que aparecen en el Cuadro 1, sumados a otras evidencias aportadas por la investigación basada en TAP, sugieren que el desarrollo de competencias argumentativas requiere de estrategias pedagógicas que las promuevan mediante un trabajo sistemático y 
sostenido a través del tiempo, pues, de otro modo, la calidad de la argumentación tiende a ser baja. Los resultados también sugieren que el uso de la argumentación puede redundar en un mejoramiento de la enseñanza y el aprendizaje de las ciencias en sus distintos niveles. Además, los investigadores parecen concordar en que el diseño de estrategias capaces de promover la argumentación en el aula, requiere comprender las características de los argumentos producidos por los estudiantes, tal como queda en evidencia al revisar los problemas abordados por diversas investigaciones realizadas durante la última década. Así por ejemplo, Jiménez-Aleixandre (2002) realizó un estudio de caso para examinar la toma de decisiones y la elaboración de argumentos respecto de temas medioambientales en estudiantes de secundaria, comparando dichos argumentos con los producidos por un experto. Berland y Reiser (2009) identificaron tres objetivos relacionados con las prácticas científicas (comprensión, articulación y persuasión) y los emplearon para estudiar cómo los estudiantes se involucran en estas prácticas, y también para diseñar ambientes de aprendizaje que permitan apoyar su aprendizaje. Naylor, Keogh y Downing (2007), preocupados por los bajos niveles de argumentación mostrados por estudiantes del Reino Unido, se propusieron investigar el grado en que los alumnos de primaria se involucran en la argumentación, como también las características de dichos argumentos.

Como se mencionó en la introducción, el estudio de la argumentación en los procesos de enseñanza y aprendizaje de las ciencias constituye una línea de investigación que ha experimentado un crecimiento significativo durante las últimas dos décadas. Dentro de esta línea de investigación, los estudios basados en TAP también han aumentado de forma importante. La Gráfica 1 muestra el número de publicaciones durante las últimas dos décadas, en tres revistas

Gráfica 1. Número de publicaciones sobre argumentación en educación en ciencias en el periodo 1991-2010

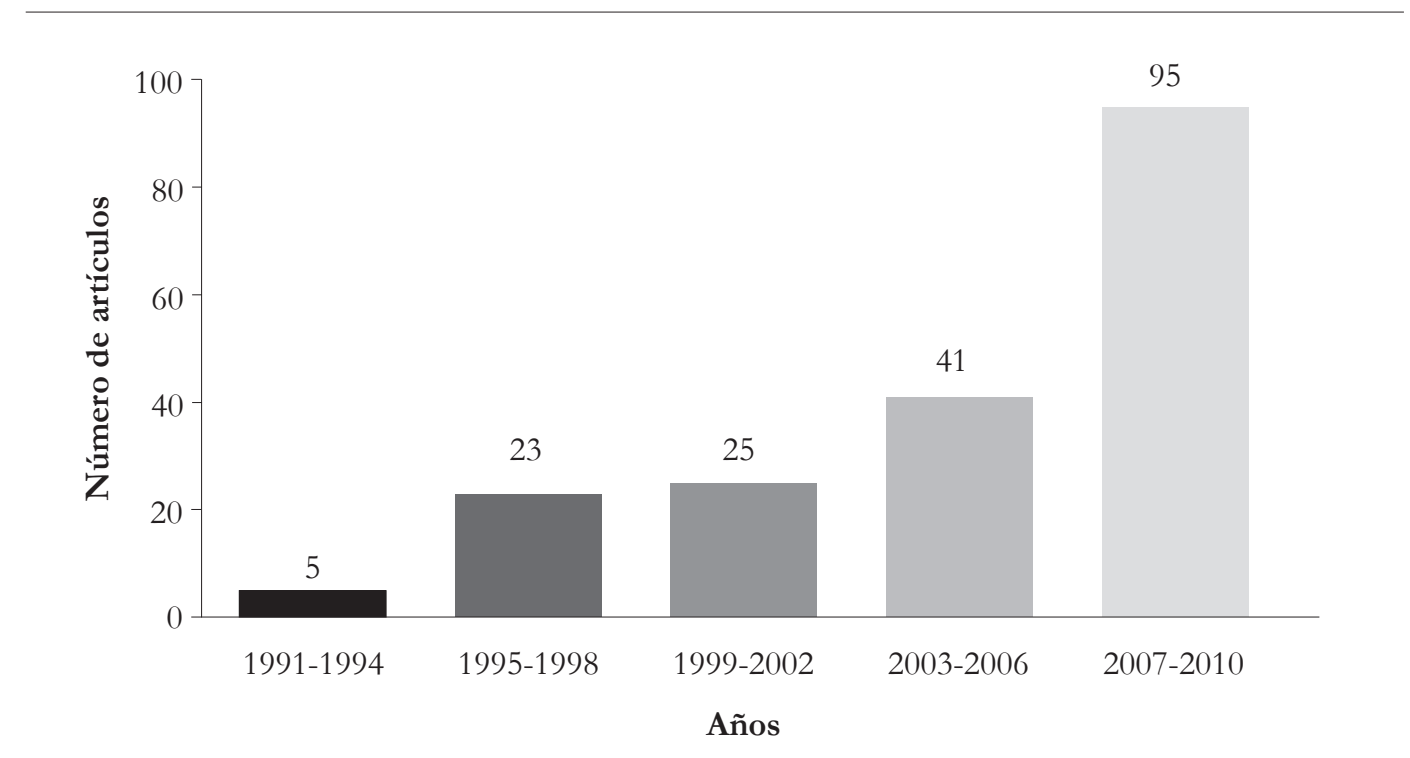

Fuente: elaboración del autor, basado en datos recuperados de Web of Knowledge (Disponible en: < http:/ /www. webofknowledge.com/>. Acceso el: 9 mayo 2011). 
especializadas en educación en ciencias que presentan los más altos índices de impacto en la Web of Knowledge, de modo que pueden considerarse representativas del panorama sobre el tema: Science Education, Journal of Research in Science Teaching, International Journal of Science Education. Al observar la gráfica, es posible apreciar el importante crecimiento que ha experimentado la literatura, especialmente durante los últimos cuatro años. Si se suma el número de publicaciones durante los primeros cuatro periodos (1991-2006), se obtiene una publicación menos que el total del último periodo (2007-2010), lo que da cuenta de la magnitud del incremento de los estudios en este campo.

Los resultados de la investigación reciente basada en TAP avalan los antecedentes de los estudios anteriores, y también aportan nuevas evidencias sobre los procesos de argumentación en las clases de ciencias (BERLAND; REISER, 2009; CAVAGNETTO, HAND; NORTON-MEIER, 2010; CLARK; SAMPSON, 2008; DAWSON; VENVILLE, 2010).

Cuando Toulmin formuló su modelo argumentativo, nunca hizo alusión a sus potenciales aplicaciones educativas. Como hemos señalado antes, sus motivaciones eran de índole estrictamente filosófica. Por este motivo, algunos investigadores en educación han considerado necesario modificar o enriquecer el modelo original, para que pueda dar cuenta de manera más adecuada de los fenómenos que ocurren en el aula. Así por ejemplo, McNeill y Krajcik (2007) han propuesto un esquema que simplifica el modelo inicial de Toulmin. Estos autores proponen un esquema que, a diferencia del original, incorpora sólo tres elementos básicos: conclusión (claim), que corresponde a la respuesta a una pregunta; evidencia (evidence), que consiste en la información o datos que apoyan la conclusión, y razón (reasoning), que corresponde a una justificación que muestra porqué la evidencia permite apoyar la conclusión. Vemos que el modelo de McNeill y Krajcik mantiene la estructura básica propuesta por Toulmin, pero la simplifica y redefine sus componentes, entre otras cosas, introduciendo la noción de razón (reasoning) para abarcar tanto las garantías como los sustentos, y empleando una noción de conclusión más acorde con una situación típica de aula, donde se generan discusiones en las cuales los estudiantes responden preguntas.

Otros autores parecen haber tomado el camino contrario, buscando ampliar algunos componentes de TAP en vez de intentar sintetizarlos, aunque ambos enfoques no son excluyentes, sino que pueden ser complementados e integrados. Un ejemplo de propuesta que busca extender los componentes originales de TAP es el trabajo de Kelly, Druker y Chen (1998) quienes han incorporado la distinción entre datos hipotéticos y datos empíricos. Los primeros pueden corresponder a información suministrada por el docente, por un libro, etc., mientras que los segundos son obtenidos a partir de la experiencia, como por ejemplo, una actividad de laboratorio. Existen otros autores que han sugerido cambios o ajustes al esquema de Toulmin (por ejemplo, BRAVO; JIMÉNEZ-ALEIXANDRE, 2009), aunque en general se trata de intervenciones menores que no han alterado la estructura básica del modelo. Sin embargo, la mayoría de los estudios que han empleado TAP han seguido los lineamientos del esquema original.

Desde un punto de vista teórico, las investigaciones basadas en TAP, así como gran parte de los estudios sobre argumentación en las aulas de ciencias, han aportado evidencias que permiten enriquecer distintos frentes de la investigación educativa que son complementarios de los trabajos sobre argumentación, tales como los estudios sobre cambio conceptual, resolución de problemas, pensamiento crítico, etc. Sin embargo, dado que la evaluación de la calidad de la argumentación es un tema central en la investigación basada en TAP, el aporte teórico que parece 
más significativo consiste precisamente en el desarrollo de potentes instrumentos que permitan efectuar dicha evaluación. Como se discutirá en otra sección de este trabajo, la evaluación del discurso argumentativo es un tema crítico que permite poner al descubierto algunas falencias de la investigación en este campo.

Desde una perspectiva práctica, los estudios basados en TAP han contribuido en diversos aspectos. Una investigadora que ha empleado de manera consistente el modelo de Toulmin, resume dichas contribuciones en los siguientes términos:

[...] permite mejorar los procesos de aprendizaje (aprender a aprender); promueve la formación de una ciudadanía responsable, capaz de participar en las decisiones sociales ejerciendo el pensamiento crítico; permite desarrollar competencias relacionadas con las formas de trabajar en la comunidad científica, y con el desarrollo de ideas sobre la naturaleza de la ciencia que hagan justicia su complejidad, es decir, la denominada cultura científica (JIMÉNEZ-ALEIXANDRE, 2010).

Aun cuando estas contribuciones no son privativas de TAP, constituyen una excelente síntesis de los potenciales aportes del modelo de Toulmin a la educación, tanto dentro como fuera del ámbito de las ciencias. Es preciso enfatizar que se trata de aportes potenciales, pues la transferencia de la investigación basada en TAP a escuelas y universidades está recién comenzando en los países desarrollados, de modo que sus efectos a gran escala aun están lejos de poder evaluarse adecuadamente.

\section{Algunas limitaciones del modelo}

Pese a la enorme influencia que TAP ha ejercido sobre la investigación educativa en ciencias, el modelo también presenta algunas limitaciones. Así, distintos investigadores han descubierto que en ocasiones resulta en extremo difícil, si no imposible, determinar qué cuenta como dato, conclusión, garantía, sustento o refutación (JIMÉNEZ-ALEIXANDRE; BUGALLO RODRÍGUEZ; DUSCHL, 2000; KELLY; DRUKER; CHEN, 1998), lo que puede generar enormes dificultades si se pretende emitir un juicio acerca de la calidad de un argumento, como ocurre frecuentemente en las investigaciones basadas en TAP.

Para captar claramente la clase de dificultades que conlleva TAP, conviene revisar nuevamente la Figura 2. Si se considera, por ejemplo, el dato y la conclusión del argumento sobre el Péndulo de Foucault, es posible advertir que bajo ciertas condiciones, los roles de ambos componentes pueden ser intercambiados. Vale decir, lo que aparece como dato: el plano de oscilación parece girar, puede considerarse una conclusión, mientras que la conclusión: la Tierra gira sobre su propio eje, puede asumir el papel de dato. Esto sugiere que las perspectivas del investigador o del educador pueden determinar los resultados derivados de la aplicación de TAP.

Las debilidades de TAP han conducido a algunos investigadores a formular modelos alternativos con el propósito específico de dar cuenta de diversos aspectos que son característicos del discurso argumentativo en las clases de ciencias (KELLY; TAKAO, 2002; LAWSON, 2003; MCNEILL, et al., 2006; SANDOVAL; 2003; ZOHAR; NEMET, 2002). Naturalmente, todos 
estos modelos presentan fortalezas y debilidades, tal como sucede con TAP. En definitiva, se trata de esquemas argumentativos que resultan idóneos bajo ciertos contextos, mientras que en otras circunstancias, el modelo de Toulmin puede presentar ventajas comparativas. Por tanto, el criterio y la experiencia del investigador, así como la naturaleza de su objeto de estudio, son los elementos clave que determinan la elección sobre uno u otro modelo.

\section{Orientaciones metodológicas}

Para tener una idea del contexto metodológico donde se ubica la investigación basada en TAP, conviene regresar al Cuadro 1 . Al efectuar un examen detallado de las investigaciones ahí consignadas, se concluye que las publicaciones más citadas presentan orientaciones metodológicas diversas que abarcan desde la investigación experimental (BELL; LINN, 2000), los estudios de caso (JIMÉNEZ-ALEIXANDRE; BUGALLO RODRÍGUEZ; DUSCHL, 2000), la investigación etnográfica (KELLY; DRUKER; CHEN, 1998), etc. Así, los estudios basados en TAP dan cabida a una amplia gama de metodologías, tanto cualitativas como cuantitativas. Sin embargo, estas dos grandes corrientes metodológicas presentan fortalezas y debilidades que es importante considerar. Por ejemplo, la gran cantidad de factores que afectan los argumentos producidos en aula, tales como el contexto disciplinario, las características de estudiantes y profesores, el marco curricular, etc., se combinan de maneras complejas, haciendo difícil generar las condiciones controladas que suelen requerir los estudios cuantitativos. Sin embargo, estos estudios permiten generalizar y comparar resultados, controlar y aislar variables, así como estudiar los efectos diferenciales de los factores implicados. Por otra parte, las investigaciones cualitativas, en especial, aquellas realizadas en condiciones naturales, permiten un examen profundo de los procesos argumentativos, lo que resulta imposible desde un enfoque experimental. No obstante, los resultados cualitativos no son generalizables ni comparables, lo que constituye un importante obstáculo si, por ejemplo, se pretende que la investigación basada en TAP o en otros modelos argumentativos, tenga impacto a nivel de políticas educativas.

Es interesante considerar la posibilidad de integrar los enfoques cualitativos y cuantitativos. Las investigaciones mixtas son más bien escasas en los estudios sobre argumentación en las clases de ciencias, aun cuando pueden presentar ventajas comparativas, tales como: alcanzar una perspectiva más amplia y profunda de la relación entre enseñanza, aprendizaje y argumentación; producir datos de mayor riqueza y variedad; apoyar con mayor solidez las conclusiones, etc. Sin embargo, los métodos mixtos no están exentos de dificultades, como por ejemplo, problemas de costo, de tiempo, y también de experticia, pues la integración de ambas metodologías impone mayores exigencias a los investigadores (HERNÁNDEZ; FERNÁNDEZ; BAPTISTA, 2010).

Pese a que TAP ha sido empleado en el marco de diversas metodologías, la investigación en este campo revela una clara preeminencia de los enfoques cualitativos (por ejemplo, JIMÉNEZ-ALEIXANDRE; BUGALLO RODRÍGUEZ; DUSCHL, 2000; KOLSTØ, 2006; VON AUFSCHNAITER et al., 2008), tal como se muestra en la Gráfica 2, donde se observa que el porcentaje (en números redondos) de investigaciones cualitativas basadas en TAP supera ampliamente a los estudios cuantitativos en este campo. Entre otras razones, esto puede explicarse al reparar en una propuesta de Deanne Kuhn (1993), según la cual, el discurso argumentativo exterioriza el razonamiento argumentativo. En otras palabras, el discurso argumentativo 
Gráfica 2. Porcentaje de publicaciones de la última década según la metodología empleada (cuantitativa $\mathrm{v} / \mathrm{s}$ cualitativa) para aquellos artículos de investigación empírica basada en TAP

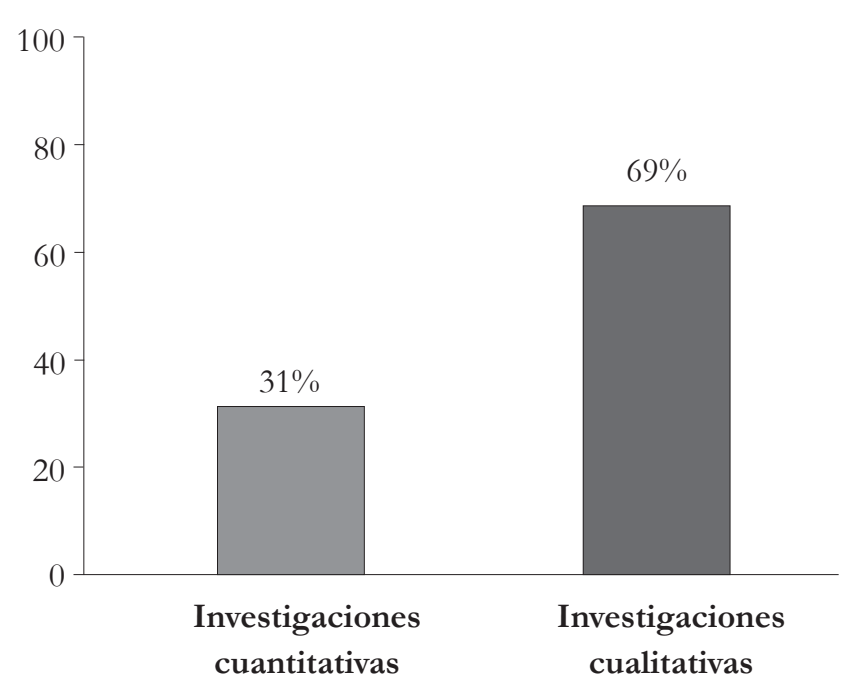

Fuente: elaboración del autor, basado en datos recuperados de Web of Knowledge (disponible en: <http://www.webofknowledge.com/>. Acceso el: 9 mayo 2011).

permite "acceder" a la mente de los estudiantes para conocer sus procesos de razonamiento. Esta propuesta define claramente un objeto de estudio - el discurso argumentativo - que por sus características, resulta idóneo para ser examinado en grupos pequeños y bajo condiciones naturales, como sucede en las clases de ciencias (por ejemplo, KELLY; DRUKER; CHEN, 1998), más que a través de respuestas obtenidas mediante mediciones estandarizadas. Sin embargo, el análisis del discurso argumentativo conlleva dificultades teórico-metodológicas que es importante considerar, y entre las cuales se cuentan: la subjetividad inherente a todo análisis interpretativo; la necesidad de trabajar con grupos pequeños, lo que exige contar con datos procedentes de diversas fuentes para aumentar la fiabilidad; la escasa disposición de algunos estudiantes a participar de los debates orales, lo que obliga a diseñar métodos especiales para recopilar información, etc. (JIMÉNEZ-ALEIXANDRE; DÍAZ DE BUSTAMENTE, 2003).

Una interesante opción metodológica que al parecer no ha sido explorada por los estudios basados en TAP, consiste en la investigación de diseño (Design Based Research). Este enfoque es usado para estudiar el aprendizaje en entornos que son diseñados y cambiados sistemáticamente por el investigador (BARAB, 2006). En otras palabras, la investigación de diseño busca estudiar de cerca un único entorno de aprendizaje, el que usualmente pasa por varios ciclos iterativos de evaluación y reevaluación mientras transcurre en contextos naturales (VAN DEN AKKER et al., 2006). Cada ciclo iterativo permite detectar falencias en el diseño, corregirlas, y arribar a un prototipo de intervención satisfactorio. El objetivo fundamental de esta aproximación metodológica consiste en generar principios de diseño prácticos y generalizables (WANG; HANNAFIN, 2005). Dadas las características de este enfoque, podría resultar adecuado para 
diseñar, revisar y evaluar estrategias y ambientes de aprendizaje basados en la argumentación, como también para refinar el marco teórico existente sobre el discurso argumentativo en las clases de ciencias. Por lo tanto, se trata de una opción metodológica que podría complementar y enriquecer la investigación basada en TAP, así como en otros modelos argumentativos.

\section{Una aproximación a las líneas de la investigación basada en TAP}

Un aspecto que llama la atención respecto de la investigación educativa basada en TAP es su marcada tendencia prescriptiva. Es decir, más allá de los objetivos, metodologías o contextos de investigación, generalmente TAP ha sido usado como un estándar para evaluar la calidad argumentativa, mientras que otros aspectos de la argumentación aparecen más bien subordinados a esta dimensión prescriptiva (por ejemplo, BELL; LINN, 2000; DRIVER; NEWTON; OSBORNE, 2000; KELLY; DRUKER; CHEN, 1998; OSBORNE; ERDURAN; SIMON, 2004; SIMON; ERDURAN; OSBORNE, 2006; VON AUFSCHNAITER et al., 2008). Para ahondar sobre este punto, conviene regresar al ejemplo sobre el péndulo de Foucault presentado en la Figura 2. Se observa que TAP consiste de seis componentes que, en contadas ocasiones, aparecen juntas para dar forma a una discusión en contextos cotidianos. Esta reflexión sugiere de inmediato la posibilidad de evaluar la calidad de un argumento en términos de los componentes de TAP que están presentes (o ausentes) en el discurso. Esta es precisamente la idea que sustenta una de las aplicaciones más difundidas e ilustrativas del modelo de Toulmin. Se trata de una escala que permite cuantificar la argumentación según cinco niveles, que van desde la más básica (nivel 1), hasta una argumentación muy sofisticada (nivel 5).

Esta escala fue propuesta por Osborne, Erduran y Simon (2004), y ha sido usada por investigadores de distintos países para evaluar los argumentos sobre ciencias desarrollados por estudiantes y profesores (para una perspectiva más amplia sobre el uso de este cuadro, ver ERDURAN; SIMON; OSBORNE, 2004). El Cuadro 2 muestra la escala con la descripción de cada uno de sus niveles. Las investigaciones realizadas hasta ahora han sido poco alentadoras, pues han mostrado escasos argumentos sobre los niveles 2 o 3 , principalmente debido a la ausencia de refutaciones. En efecto, como revelaron las investigaciones pioneras de Kuhn (1991), la capacidad de emplear refutaciones es una de las habilidades de mayor complejidad.

Regresando el comienzo de esta sección, es importante enfatizar que el Cuadro 2 ilustra claramente el carácter prescriptivo de la investigación basada en TAP, pues pone en evidencia que la emisión de juicios acerca de la calidad del discurso argumentativo es un aspecto que emerge de manera natural a partir del modelo de Toulmin. Una interesante excepción a esta tendencia prescriptiva en el uso de TAP, es un estudio de Kolstø (2006) que analiza los argumentos de un grupo de estudiantes enfrentados a un problema sociocientífico, es decir, un problema que si bien requiere de conocimientos científicos para ser abordado, se refiere a temas de interés ciudadano que generan controversia y debate social (KOLSTØ; RATCLIFFE, 2008), lo que marca una importante diferencia respecto de los problemas científicos, que están circunscritos a temáticas que son propias y específicas de la ciencia. Hay que señalar que no siempre resulta fácil decidir si un argumento apunta a problemas científicos o sociocientíficos, aun cuando con fines investigativos es frecuente efectuar esta distinción. El problema sociocientífico estudiado por Kolstø se refiere a la construcción de un tendido eléctrico, y la posibilidad de que ello au- 
Cuadro 2. Niveles de argumentación

\begin{tabular}{|c|l|}
\hline Niveles & \multicolumn{1}{|c|}{ Descripción } \\
\hline 1 & $\begin{array}{l}\text { Argumentación que consisten de argumentos que son conclusiones simples versus contra } \\
\text { conclusiones o conclusiones versus conclusiones. }\end{array}$ \\
\hline 2 & $\begin{array}{l}\text { Argumentación que tiene argumentos que consisten en conclusiones, datos, garantías o } \\
\text { sustentos, pero no contiene ninguna refutación. }\end{array}$ \\
\hline 3 & $\begin{array}{l}\text { Argumentación que tiene argumentos con una serie de conclusiones o contra } \\
\text { conclusiones con cualquier dato, garantías, o sustentos con refutaciones débiles } \\
\text { ocasionales. }\end{array}$ \\
\hline 4 & $\begin{array}{l}\text { Argumentación que muestra argumentos con una conclusión que tiene una refutación } \\
\text { claramente identificable. }\end{array}$ \\
\hline 5 & \begin{tabular}{l} 
Argumentación que manifiesta un amplio argumento con más de una refutación. \\
\hline
\end{tabular} \\
\hline
\end{tabular}

Fuente: Traducida y adaptada de Osborne, Erduran y Simon (2004).

mente el riesgo de leucemia en los niños. Enfrentados a este problema, los argumentos de los estudiantes fueron clasificados en cinco categorías, las cuales, según el autor, podrían posibilitar que los estudiantes y profesores de ciencias argumenten y tomen decisiones de manera más reflexiva. En el contexto de TAP, el trabajo de Kolstø puede ser clasificado como un estudio descriptivo, en contraste con los estudios prescriptivos presentados antes. La escasez de estudios descriptivos es un tema relevante sobre el cual profundizaremos en la sección final de este artículo, pues genera diversas reflexiones e interrogantes respecto de la utilización del modelo de Toulmin en la investigación sobre los procesos de enseñanza y aprendizaje de las ciencias.

Se ha señalado que el modelo de Toulmin no es el único esquema empleado por los especialistas en educación en ciencias. Así, TAP está inserto en una línea de investigación amplia, que presenta diferentes focos o líneas específicas de estudio, que incluyen como caso particular los trabajos basados en TAP. Dentro de estas líneas específicas, resulta particularmente relevante distinguir entre los contextos científicos y sociocientíficos, pues éstos abarcan la inmensa mayoría de la investigación educativa sobre argumentación en ciencias. Además, en el marco de TAP, la distinción entre estos contextos resulta crucial, dada la dependencia del discurso argumentativo respecto del campo, tal como enfatiza el modelo de Toulmin.

Los problemas científicos y sociocientíficos corresponden a campos de discurso diferentes, pues, aun cuando ambos se relacionan con contenidos propios de la ciencia, la perspectiva frente a estos contenidos es muy diferente, y las temáticas involucradas en la discusión también son distintas, tal como se ha señalado un poco atrás. Además, los problemas científicos son valóricamente neutros, es decir, su resolución está circunscrita a un dominio técnico, mientras que los sociocientíficos poseen una importante carga valórica, ya que admiten soluciones no técnicas, basadas en apreciaciones personales y en escalas de valores que pueden variar considerablemente de una persona a otra. Por tanto, la distinción entre ambos contextos se justifica 
desde un punto de vista teórico, pero también se justifica empíricamente, ya que los estudios sugieren que en contextos sociocientíficos, los estudiantes discuten de manera diferente que cuando lo hacen acerca de asuntos científicos convencionales (JIMÉNEZ-ALEIXANDRE; PEREIRO MUNOZ; AZNAR CUADRADO, 2000).

También es posible encontrar problemas cuyo campo de discurso se sitúa en un punto intermedio entre las cuestiones científicas y sociocientíficas, pero en este apartado nos interesa dar cuenta de los principales contextos generados por los investigadores, antes que atender a las situaciones cotidianas de aula, donde las discusiones pueden presentar complejos matices. Los resultados de las investigaciones en esta línea son variados, y han arrojado luz sobre diversos aspectos del discurso argumentativo (DAWSON; VENVILLE, 2010; SEETHALER; LINN, 2004; TOPCU; SADLER; YILMAZ-TUZUN, 2010).

La distinción, por una parte, entre contextos científicos y sociocientíficos, y, por otra, entre estudios descriptivos y prescriptivos, permite sugerir un esquema con las grandes líneas de la investigación basada en TAP, tal como muestra la Figura 3. Como es evidente, también es posible identificar otros focos de estudio, los cuales, desde la perspectiva de este artículo, no constituyen líneas de investigación propiamente dichas, pues se trata de temáticas específicas que no generan distinciones de importancia dentro del modelo de Toulmin. Así por ejemplo, existen estudios que han examinado los procesos de argumentación en el contexto de las tecnologías de la información y de la comunicación, (BELL; LINN, 2000; CLARK; SAMPSON, 2007, 2008; CROSS et al., 2008), otros que se han orientado al estudio de las prácticas docentes (MCNEILL, 2009; SCHOLTZ et al., 2008; SIMON; OSBORNE; ERDURAN, 2003), etc.

Figura 3. Una visión esquemática de las grandes líneas de la investigación basada en TAP

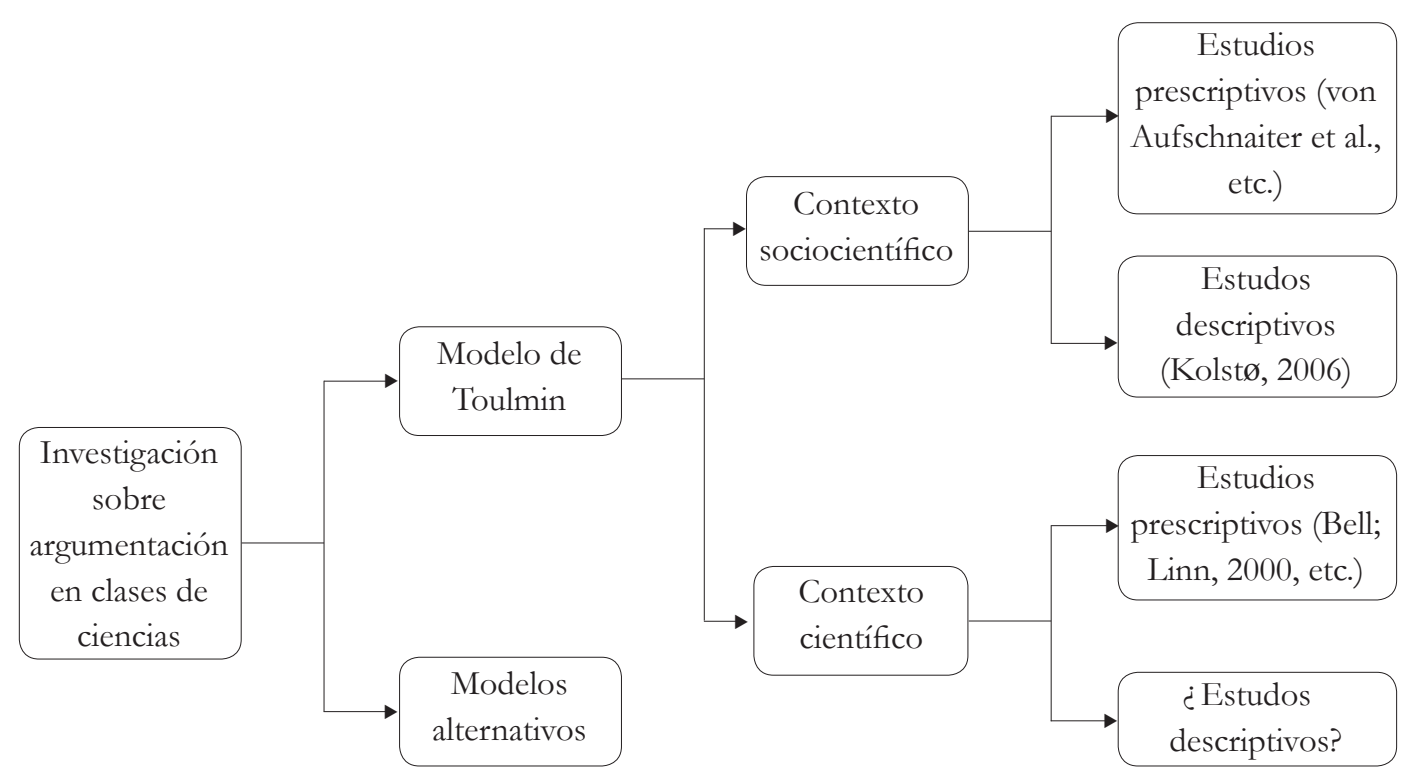

Fuente: elaboración del autor. 


\section{Una mirada crítica a la investigación basada en TAP}

\section{¿Razonar o argumentar?}

El libro con el que Toulmin (1958) da origen a la teoría moderna de la argumentación se titula: Los usos del argumento ${ }^{5}$. Un libro posterior, que aborda la misma temática pero desde una perspectiva menos formal, se titula: Introducción al razonamiento ${ }^{6}$ (TOULMIN; RIEKE; JANIK, 1979), lo que sugiere que los términos argumento y razonamiento son empleados como equivalentes o intercambiables, cuando en estricto rigor se trata de nociones distintas. Esta misma falta de precisión se observa en un porcentaje importante de la investigación basada en TAP (ver, por ejemplo, JIMÉNEZ-ALEIXANDRE; BUGALLO RODRÍGUEZ; DUSCHL, 2000; KELLY; DRUKER; CHEN 1998; OSBORNE; ERDURAN; SIMON, 2004).

Para aclarar esta última afirmación es necesario consensuar que la noción de razonamiento alude a procesos mentales. En un sentido restringido pueden distinguirse distintos tipos de razonamiento. El que aquí importa es el denominado razonamiento argumentativo, y que en la perspectiva de Deanna Kuhn puede entenderse como una actividad mental que se corresponde con la actividad discursiva de argumentar. En otras palabras, el discurso argumentativo exterioriza el razonamiento argumentativo (KUHN, 1993). Así, razonamiento y argumentación se encuentran íntimamente ligados, aun cuando en estricto rigor no son equivalentes. La investigación basada en TAP suele tratar estas nociones como equivalentes o intercambiables, sin definir claramente el concepto de razonamiento, sin establecer su relación con la argumentación, y, generalmente, sin siquiera introducir la distinción entre razonamiento y razonamiento argumentativo.

Estas imprecisiones semánticas tienen más relevancia de lo que puede parecer en primera instancia, pues las nociones de razonamiento y argumentación no solo tienen distinto estatus ontológico sino también epistemológico. En efecto, las investigaciones empíricas sobre razonamiento trabajan con entidades no observables, definidas operacionalmente en base a marcos teóricos sobre procesos mentales, mientras que la investigación basada en TAP trabaja con entidades observables (discursos) definidas en base a modelos argumentativos cuya fundamentación es filosófica y no psicológica. Por lo tanto, no es posible tratar las nociones de razonamiento y argumentación como equivalentes o intercambiables sin antes justificar dicha equivalencia. Vale decir, siempre que se emplee la noción de razonamiento, es necesario definirla claramente, justificando su relación y pertinencia con el modelo argumentativo utilizado, ya sea el esquema de Toulmin o cualquier otro. Corregir estas imprecisiones puede contribuir a mejorar la comunicación, la comprensión y la comparación de los resultados de la investigación en este campo.

\footnotetext{
${ }^{5}$ En el original The uses of argument.

${ }^{6}$ En el original An introduction to reasoning.
} 


\section{Investigación prescriptiva versus descriptiva}

Los investigadores parecen concordar en que la principal fortaleza de TAP reside en su capacidad para evaluar la argumentación. Así, la investigación basada en TAP ha realizado importantes contribuciones al conocimiento del perfil y la calidad del discurso argumentativo en las clases de ciencias. Sin embargo, hasta cierto punto, esta fortaleza ha derivado en una debilidad, que se traduce en una marcada tendencia prescriptiva, y en un escaso interés por la investigación descriptiva.

La importancia de emplear el modelo de Toulmin para estudiar el discurso argumentativo desde un punto de vista descriptivo, queda de manifiesto al examinar el trabajo realizado por Kolstø (2006). Como se ha señalado antes, este autor caracterizó cinco tipos de argumentos empleados por los estudiantes para discutir sobre cuestiones sociocientíficas. Kolstø sugiere que dicha caracterización podría ser utilizada en las clases de ciencias para: posibilitar la toma de decisiones reflexivas sobre temas de interés ciudadano relacionados con la ciencia; explorar la pertinencia de diferentes tipos de conocimiento; poner en evidencia que existen distintas maneras de llegar a una conclusión; generar conciencia respecto de la importancia de incorporar más información al debate que la considerada inicialmente; caracterizar los argumentos propios para tomar conciencia de sus fortalezas y debilidades, así como de la existencia de argumentos alternativos, etc. Estas sugerencias son elocuentes, y muestran que la investigación descriptiva puede constituirse en una línea de estudio capaz de aportar importantes resultados. Otra elemento de reflexión que surge de la investigación de Kolstø, es que las dimensiones descriptivas y prescriptivas no son excluyentes sino complementarias, lo que abre interesantes perspectivas a la investigación basada en TAP.

\section{Reflexiones finales: argumentando a favor de la argumentación}

A través de la presente revisión se ha constatado que la investigación basada en TAP posee una sólida fundamentación teórica, una orientación metodológica amplia, y una relevancia educativa que, a la luz de los resultados, perece innegable. Además, la investigación en este campo ha experimentado un crecimiento significativo durante la última década, lo que da cuenta de la importancia que los especialistas le asignan al estudio de la argumentación en las clases de ciencias. Sin embargo, además de señalar las bondades de este campo, también se han descrito algunas falencias y problemas abiertos que, lejos de representar obstáculos, brindan múltiples oportunidades para emprender futuros estudios.

La investigación basada en TAP no sólo es relevante porque ha mostrado que la argumentación permite mejorar el aprendizaje de las ciencias. Desde una perspectiva amplia, también es relevante porque a través de la argumentación, los estudiantes se introducen en la cultura de la ciencia, pues, como ya se ha mencionado, el conocimiento científico es construido, comunicado y evaluado mediante la argumentación. Así, más que un cúmulo de resultados teóricos y experimentales, la ciencia es una forma crítica y reflexiva de estudiar la naturaleza, es decir, es una forma argumentada de entender el mundo. Los contenidos de la ciencia cambian con el tiempo, pero la forma en que dichos contenidos son construidos, comunicados y evaluados permanece sin cambios. Para que los estudiantes adquieran una alfabetización científica que 
les permita desenvolverse con éxito en la sociedad del conocimiento, el aprendizaje significativo parece insuficiente; también se requiere promover la argumentación como una dimensión fundamental de la educación científica en todos sus niveles. Sin embargo, es importante tener presente que el modelo de Toulmin, pese a su innegable protagonismo, es sólo uno de entre los diversos esquemas argumentativos que los especialistas tienen a su disposición. Optar por uno u otro enfoque es una cuestión de conveniencia, que marca diferencias de forma y no de fondo, pues más allá del modelo argumentativo utilizado, el objeto de estudio es el mismo: la argumentación en las clases de ciencias; y la meta también es una sola: entregar a los estudiantes una educación cientifica de calidad.

\section{Referencias}

BARAB, S. A. Design-based research: a methodological toolkit for the learning sciences. In: SAWYER, K. (Ed.). The Cambridge handbook of the learning sciences. Cambridge: Cambridge University Press, 2006. p. 153-169.

BELL, P.; LINN, M. C. Scientific arguments as learning artifacts: designing for learning from the web with KIE. International Journal of Science Education, Abingdon, v. 22. n. 8, p. 797-817, 2000. Disponible en: < http://www.designbasedresearch.org/reppubs/bell-Linn. pdf>. Acceso el: 19 en. 2015.

BERLAND, L. K.; REISER, B. J. Making sense of argumentation and explanation. Science Education, Hoboken, v. 93, n.1, p. 26-55, 2009.

BRAVO, B.; JIMÉNEZ-ALEIXANDRE, M. P. ¿Criamos leones en granjas? Uso de pruebas y conocimiento conceptual en un problema de acuicultura. In: CONGRESO INTERNACIONAL SOBRE INVESTIGACIÓN EN LA DIDÁCTICA DE LAS CIENCIAS, 8., 2009, Barcelona. Enseñanza de las Ciencias, Barcelona, 2009. Número extra.

CAVAGNETTTO, A.; HAND, B. M.; NORTON-MEIER, L. The nature of elementary Student science discourse in the context of the science writing heuristic approach.

International Journal of Science Education, Abingdon, v. 32, n. 4, p. 427-449, 2010.

CLARK, D. B.; SAMPSON, V. Assessing dialogic argumentation in online environments to relate structure, grounds, and conceptual quality. Journal of Research in Science Teaching, Hoboken, v. 45, n. 3, p. 293-321, 2008.

Personally-seeded discussions to scaffold online argumentation. International

Journal of Science Education, Abingdon, v. 29, n. 3, p. 253-277, 2007.

CROSS, D. et al. Argumentation: a strategy for improving achievement and revealing scientific identities. International Journal of Science Education, Abingdon, v. 30, n. 6, p. $837-861,2008$. 
El modelo argumentativo de Toulmin ...

DAWSON, V. M.; VENVILLE, G. Teaching strategies for developing students’ argumentation skills about socioscientific issues in high school genetics. Research in Science Education, Dordrecht, v. 40, n. 2, p. 133-148, 2010.

DRIVER, R.; NEWTON, P.; OSBORNE, J. Establishing the norms of scientific argumentation in classrooms. Science Education, Hoboken, v. 84, n. 3, p. 287-312, 2000.

ERDURAN, S.; SIMON, S.; OSBORNE, J. TAPping into argumentation: developments in the application of Toulmin's argument pattern for studying science discourse. Science Education, Hoboken, v. 88, n. 6, p. 915-933, 2004.

HART, C. Doing a literature review: releasing the social science research imagination. London: Sage, 1998.

HERNÁNDEZ, R.; FERNÁNDEZ, C.; BAPTISTA, P. Metodología de la investigación. 5. ed. México: McGraw-Hill Interamericana, 2010.

JIMÉNEZ-ALEIXANDRE, M. P. 10 ideas clave: competencias en argumentación y uso de pruebas. Barcelona: Graó, 2010.

. Knowledge producers or knowledge consumers?: argumentation and decision making about environmental management. International Journal of Science Education, Abingdon, v. 24, n. 11, p. 1171-1190, 2002.

JIMÉNEZ-ALEIXANDRE, M. P.; DÍAZ DE BUSTAMENTE, J. Discurso de aula y argumentación en la clase de ciencias: cuestiones teóricas y metodológicas. Enseñanza de las Ciencias, Barcelona, v. 21, n. 3, p. 359-370, 2003.

JIMÉNEZ-ALEIXANDRE, M. P.; PEREIRO MUNOZ, C.; AZNAR CUADRADO, V. Promoting reasoning and argument about environmental issues. In: ANDERSON, B. et al. (Ed.). Research in didaktik of biology: proceedings of the Second Conference of European Researchers in Didaktik of Biology (ERIDOB). Göteborg: IPD, 2000. p. 215-230.

JIMÉNEZ-ALEIXANDRE, M. P.; BUGALLO RODRÍGUEZ, A. B.; DUSCHL, R. A. "Doing the lesson" or "doing Science": argument in high school genetics. Science Education, Hoboken, v. 84, n. 6, p. 757-792, 2000.

KELLY, G. J.; DRUKER, S.; CHEN, C. Students' reasoning about electricity: combining performance assessments with argumentation analysis. International Journal of Science Education, Abingdon, v. 20, n. 7, p. 849-871, 1998.

KELLY, G. J.; TAKAO, A. Epistemic levels in argument: an analysis of university oceanography students' use of evidence in writing. Science Education, Hoboken, v. 86, n. 3, p. 314-342, 2002.

KOLST $\varnothing$, S. D. Patterns in students' argumentation confronted with a risk-focused socio-scientific issue. International Journal of Science Education, Abingdon, v. 28, n. 14, p. 1689-1716, 2006.

KOLSTØ, S. D.; RATCLIFFE, M. Social aspects of argumentation. In: ERDURAN, S.; JIMÉNEZ-ALEIXANDRE, M. P. (Ed.). Argumentation in science education: perspectives from classroom-based research. Dordrecht: Springer, 2008. p. 117-136. 
KRUMMHEUER, G. The ethnography of argumentation. In: COBB, P.; BAUERSFELD, $\mathrm{H}$. (Ed.). The emergence of mathematical meaning: interaction in classroom cultures. Hillsdale: Lawrence Erlbaum, 1995. p. 229-269. (Studies in mathematical thinking and learning series).

KUHN, D. Science as argument: implications for teaching and learning scientific thinking. Science Education, Hoboken, v. 77, n. 3, p. 319-337, 1993.

. The skills of argument. Cambridge: Cambridge University Press, 1991.

. Thinking as argument. Harvard Educational Review, Cambridge, v. 62, n. 2, p. $155-178,1992$.

LAWSON, A. E. The nature and development of hypothetico-predictive argumentation with implications for science teaching. International Journal of Science Education, Abingdon, v. 25, n. 11, p. 1387-1408, 2003.

MCNEILL, K. L. Teachers' use of curriculum to support students in writing scientific arguments to explain phenomena. Science Education, Hoboken, v. 93, n. 2, p. 233-268, 2009.

MCNEILL, K. L.; KRAJCIK, J. Middle school students' use of appropriate and inappropriate evidence in writing scientific explanations. In: LOVETT, M.; SHAH, P. (Ed.). Thinking with data. New York: Taylor \& Francis, 2007. p. 233-265.

. Synergy between teacher practices and curricular scaffolds to support students in using domain-specific and domain-general knowledge in writing arguments to explain phenomena. The Journal of the Learning Sciences, Philadelphia, v. 18, n. 3, p. 416-460, 2009.

MCNEILL, K. L. et al. Supporting students' construction of scientific explanations by fading scaffolds in instructional materials. Journal of the Learning Sciences, Philadelphia, v. 15 , n. 2, p. 153-191, 2006.

MITCHELL, S. Improving the quality of argument in higher education: interim report. London: Middlesex University, School of Education, 1996.

NAYLOR, S.; KEOGH, B.; DOWNING, B. Argumentation and primary science. Research in Science Education, Dordrecht, v. 37, n. 1, p. 17-39, 2007.

OSBORNE, J.; ERDURAN, S.; SIMON, S. Enhancing the quality of argumentation in school science. Journal of Research in Science Teaching, Hoboken, v. 41, n. 10, p. 994-1020, 2004.

PERELMAN, C.; OLBRECHTS-TYTECA, L. Traité de l'árgumentation: la nouvelle rhétorique. Paris: Presses Universitaires de France, 1958.

PONTECORVO, C.; GIRARDET, H. Arguing and reasoning in understanding historical topics. Cognition and Instruction, Philadelphia, v. 11, n. 3, p. 365-395, 1993.

SANDOVAL, W. A. Conceptual and epistemic aspects of students' scientific explanations. Journal of the Learning Sciences, Philadelphia, v. 12, n. 1, p. 5-51, 2003. 
SCHOLTZ, Z. et al. South African teachers' ability to argue: the emergence of inclusive argumentation. International Journal of Educational Development, Oxford, v. 28, n. 1, p. 21-34, 2008.

SCHWARZ, B. B. et al. Construction of collective and individual knowledge in argumentative activity. Journal of the Learning Sciences, Philadelphia , v. 12, n. 2, p. 219-256, 2003.

SEETHALER, S.; LINN, M. Genetically modified food in perspective: an inquiry-based curriculum to help middle school students make sense of tradeoffs. International Journal of Science Education, Abingdon, v. 26, n. 14, pp. 1765-1785, 2004.

SIMON, S.; ERDURAN, S.; OSBORNE, J. Learning to teach argumentation: research and development in the science classroom. International Journal of Science Education, Abingdon , v. 28, n. 2, p. 235-260, 2006.

SIMON, S.; OSBORNE, J.; ERDURAN, S. Systemic teacher development to enhance the use of argumentation in school science activities. In: WALLACE, J.; LOUGHRAN, J. (Ed.). Leadership and professional development in science education: new possibilities for enhancing teacher learning. London: Routledge Falmer, 2003. p. 198-217.

TOPCU, M. S.; SADLER, T. D.; YILMAZ-TUZUN, O. Preservices Teachers' informal reasoning about socioscientific issues: the influence of issue context. International Journal of Science Education, Abingdon, v. 32, n. 18, p. 2475-2495, 2010.

TOULMIN, S. The uses of argument. Cambridge: Cambridge University Press, 1958.

TOULMIN, S.; RIEKE, R.; JANIK, A. An introduction to reasoning. New York: Macmillan, 1979.

VAN DEN AKKER, J. et al. Introducing educational design research. In: Educational design research. Abingdon: Routledge, 2006. p. 3-7. et al. (Ed.).

VON AUFSCHNAITER, C. et al. Arguing to learn and learning to argue: case studies of how students' argumentation relates to their scientific knowledge. Journal of Research in Science Teaching, Hoboken, v. 45, n. 1, p. 101-131, 2008.

WALTON, D. Argumentation schemes for presumptive reasoning. Mahwah: Lawrence Erlbaum, 1996.

WANG, F.; HANNAFIN, M. J. Design-based research and technology-enhanced learning environments. Educational Technology Research and Development, New York, v. 53, n. 4, p. 5-23, 2005.

ZOHAR, A.; NEMET, F. Fostering students' knowledge and argumentation skills through dilemmas in human genetics. Journal of Research in Science Teaching, Hoboken, v. 39, n. 1, p. 35-62, 2002. 